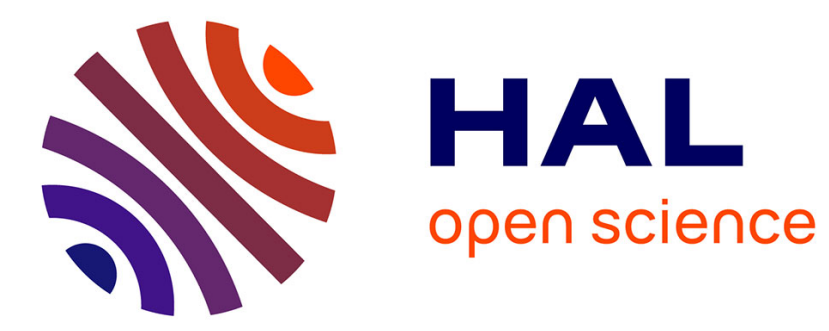

\title{
Les enjeux de la recherche en psychologie de la santé
}

\author{
Michèle Koleck, Marie Préau, Lionel Dany, Stéphane Faury
}

\section{To cite this version:}

Michèle Koleck, Marie Préau, Lionel Dany, Stéphane Faury. Les enjeux de la recherche en psychologie de la santé. Le Journal des psychologues, 2017. hal-01648420

\section{HAL Id: hal-01648420 \\ https://hal-amu.archives-ouvertes.fr/hal-01648420}

Submitted on 26 Nov 2017

HAL is a multi-disciplinary open access archive for the deposit and dissemination of scientific research documents, whether they are published or not. The documents may come from teaching and research institutions in France or abroad, or from public or private research centers.
L'archive ouverte pluridisciplinaire HAL, est destinée au dépôt et à la diffusion de documents scientifiques de niveau recherche, publiés ou non, émanant des établissements d'enseignement et de recherche français ou étrangers, des laboratoires publics ou privés. 
${ }^{1}$ Maître de conférences en psychologie différentielle de la santé, Université de Bordeaux

${ }^{2}$ Professeur de psychologie sociale de la santé, Université Lyon 2

${ }^{3}$ Professeur de psychologie sociale, Aix Marseille Université

${ }^{4}$ Psychologue, doctorante en psychologie de la santé, Université de Bordeaux

\section{Les enjeux de la recherche en psychologie de la santé}

Les enjeux de la recherche en psychologie de la santé ont évolué ces dernières années. Ils peuvent être organisés en six axes, dans lesquels coexistent à la fois recherche fondamentale et recherche appliquée qui sont explorées à travers différentes méthodes et techniques.

La recherche en psychologie de la santé est particulièrement développée en France où elle est menée par différents acteurs, à la fois des praticiens et des chercheurs (enseignants-chercheurs, doctorants et cliniciens), dans les milieux hospitalier, associatif,... Ses résultats peuvent avoir des implications pour les psychologues comme pour tous les professionnels exerçant dans le champ de la santé. Nous aborderons, dans un premier temps, les grands axes actuels de la recherche en psychologie de la santé. Dans un second temps, nous proposerons quelques exemples de recherche.

\section{Les grands axes actuels de la recherche en psychologie de la santé}

Traditionnellement, la recherche en psychologie de la santé s'articule autour de trois grands axes (cf. Fisher, 2002 ; Matarazzo, 1980 ; Ogden, 2008 ; Sarafino, 1990 ; Taylor, 1991) : (1) expliquer et proposer des voies de changement concernant les comportements de santé et les styles de vie « sains »; (2) expliquer et prédire le rôle des facteurs bio-psycho-sociaux sur la santé ; (3) éclairer et analyser les situations de maladie. Pour résumer, la recherche en psychologie de la santé met l'accent sur le rôle des facteurs psychologiques dans les causes, la progression et les conséquences de la santé et de la maladie. Nous présenterons successivement chacun de ces axes en proposant trois axes complémentaires qui, tout en entretenant des liens étroits pour certains avec les trois axes généraux traditionnels, permettent toutefois d'éclairer des enjeux plus actuels ou spécifiques concernant la recherche en psychologie de la santé. Ces trois axes complémentaires concernent la recherche en psychologie de la santé comme moyen pour (4) aider à la décision dans le champ de la santé et de la maladie ; (5) susciter la reconnaissance et l'utilisation des différents types de savoirs associés à la santé et à la maladie ; (6) questionner de façon critique les fondements et objectifs de cette discipline. 
(1) Comprendre et viser le changement aux niveaux des comportements de santé

Plusieurs théories ou modèles ont été développés au cours de ces dernières décennies avec pour objectif d'expliquer les comportements de santé : le Health Belief Model (Rosenstock, 1974), la théorie de la motivation à se protéger (Rogers, 1975), la théorie de l'action raisonnée (Ajzen, Fishbein, 1980), la théorie du comportement planifié (Ajzen, 1991) ou encore le modèle des médiateurs de la santé (Rutter, Quine, 2000). La théorie du comportement planifié, par exemple, met l'accent sur la notion d'intention comportementale (« un plan d'action visant à atteindre un but»). Cette intention comportementale est le résultat de construits (attitude envers le comportement, normes subjectives, contrôle comportemental perçu) qui sont eux-mêmes la synthèse de l'univers de croyances d'un individu à propos d'un comportement. Ces construits déterminent l'intention qui, à son tour, va être le déterminant du comportement. Le point commun de tous ces modèles est de considérer les comportements de santé comme des comportements sociaux qui sont conçus schématiquement comme étant fonction des attitudes et des croyances des individus et des groupes par rapport à des objets ou des situations (Sarafino, 1990). Les travaux relatifs à cet axe sont très nombreux car ils contribuent à répondre à une demande sociale forte concernant l'enjeu du changement des comportements de santé. Ils permettent également d'envisager les questionnements liés au champ de la prévention auprès de populations variées comme à celui de la prise en charge et des situations de maladie.

\section{(2) Prédire les issues de santé sur le plan bio-psycho-social}

Un autre axe particulièrement important dans la recherche en psychologie de la santé concerne la prédiction des issues de santé, autrement dit, l'analyse de la contribution des variables psychosociales à l'état de santé (la santé étant entendue dans ses différentes acceptions : physique, psychologique et sociale). Les modèles explicatifs, multifactoriels et interactionnistes développés dans le champ de la psychologie de la santé ont constitué des «guides » importants pour le développement de la recherche au sein de cette discipline, et ce, depuis plus de quarante ans. Les travaux sur le stress et le développement de modèles transactionnels (Lazarus, Folkman, 1984) constituent un exemple typique de cet apport. Ces modèles ont conduit à considérer comme essentielles les tentatives des individus (leurs réponses) pour s'ajuster aux perturbations - réelles et perçues - de l'environnement mettant l'accent sur la nécessité d'explorer le rôle des stratégies d'ajustement (coping) aux situations aversives (Bruchon-Schweitzer, Quintard, 2001 ; Koleck, Bruchon-Schweitzer, Bourgeois, 2003). Le modèle transactionnel, intégratif et multifactoriel (TIM ; Bruchon-Schweitzer, Dantzer, 1994 ; Bruchon-Schweitzer, Boujut, 2014) constitue un modèle intéressant dans cette perspective. Il prend en compte le rôle des facteurs transactionnels entre les antécédents (sociodémographiques, environnementaux, biomédicaux, psychosociaux, traits de 
personnalités) et les issues en terme de santé (équilibre ou état bio-psycho-social vers lequel tend l'individu, compte tenu de ses antécédents et suite à la mise en place des mécanismes transactionnels). Les nombreux travaux développés sur la qualité de vie constituent également un exemple pertinent pour illustrer cet axe de recherche particulièrement fécond de la psychologie de la santé (e.g., Fischer, Tarquinio, 2010 ; Préau, Morin, 2005). Ces approches se conjuguent avec des approches épidémiologiques tout en tentant par exemple d'appréhender plus finement ce qui peut se jouer derrière l'un des enjeux majeurs que constituent les inégalités sociales de santé. La recherche interventionnelle en psychologie de la santé peut s'inscrire dans cet axe (bien qu'elle entretienne également des enjeux avec l'axe 1). Elle vise à modifier, maintenir, stabiliser l'état interne et/ou externe du sujet par la mise en place d'une intervention (Fischer, Tarquinio, 2014). Citons, par exemple, l'évaluation des interventions de type psychoéducatif auprès des patients présentant des troubles mentaux sur leur observance thérapeutique ou encore l'évaluation des thérapies de groupe de type soutien-expression sur l'ajustement psychologique des malades du cancer. D'autres approches permettent également de co-construire avec les populations concernées des formes d'accompagnement pour des décisions complexes, comme par exemple la révélation de sa séropositivité (Préau, Beaulieu-Prévost, Henry, Veillette-Bourbeau, Otis, 2015).

\section{(3) Eclairer et analyser les situations de maladie}

L'espace du soin et de la maladie est traversé par des enjeux particulièrement prégnants qui mettent en lumière les conditions de mise en relation des acteurs de la situation thérapeutique. Un enjeu essentiel de la recherche porte, par exemple, sur la communication au sein de la relation de soins (e.g., Brashers et Goldsmith, 2009 ; Corraze, 1992 ; Morin, 2004 ; Muñoz Sastre, Mullet, Sorum, 2011). La recherche sur les composantes relationnelles/communicationnelles en psychologie de la santé peut avoir pour fonction d'éclairer les mécanismes qui concourent à la définition et la régulation des échanges d'informations en situations de soins. La recherche peut également conduire à l'élaboration de savoirs qui permettent d'accompagner les professionnels vers une analyse réflexive sur leur propre engagement dans l'action par une explicitation de leurs pratiques et des logiques sous-jacentes à leur mise en œuvre en se centrant en particulier sur l'influence des savoirs qui peuvent intervenir dans ces pratiques (Brashers, Goldsmith, 2009). Cet axe de recherche concerne également la mise à jour et la construction de modèles d'intelligibilités des situations de maladies et de soins en prenant en compte la façon dont les individus élaborent du savoir sur la maladie et sa prise en charge (cf. Pédinielli, 1999 ; Petrie, Weinman, 1997 ; Radley, 1994). Cet axe vise ainsi la mise en évidence des « grilles de lecture » que les acteurs (patients comme soignants) assignent à la situation et à ses composantes, le questionnement des enjeux identitaires (entendus comme corrélats de l'inscription et de la participation sociales des individus) et l'explication de 
comment s'opère la «maîtrise » des situations de maladie et de soins à un niveau cognitif, social, émotionnel, relationnel et comportemental (e.g., Leroy, Fournier, Penel, Christophe, 2016 ; Tosello, Dany, Le Coz, Gire, Vriet-Ndour, Einaudi, 2014). Cet axe de recherche peut être mis en relation avec certains objectifs de la recherche-action qui vise conjointement la production de connaissances au sujet d'une réalité sociale (e.g., compréhension des processus psycho-sociaux et logiques d'action) et la transformation de la réalité sociale par les différents acteurs concernés (e.g., modifications des pratiques) ou de la recherche interventionnelle. Dans cette optique, la recherche communautaire $^{1}$ en psychologie de la santé permet de questionner des problématiques identifiées, puis co-construites avec des populations sensibles ou à risque (Morin, Terrade, Préau, 2012).

\section{(4) Aider à la décision dans le champ de la santé et de la maladie}

Les savoirs produits dans le cadre des recherches en psychologie de la santé visent également à orienter les pratiques professionnelles et constituent, de fait, des aides à la décision dans le champ de la santé (e.g., décision thérapeutique, orientation des politiques de santé publique, identification des processus et mécanismes pertinents, ...). Les outils, concepts et théories mobilisés doivent permettre un éclairage suffisamment pertinent pour guider le choix vis-à-vis des options disponibles, qu'il s'agisse de choix thérapeutiques ou d'actions auprès des populations. La prévention du risque constitue, à bien des égards, un exemple quasi-prototypique pour illustrer l'apport issu des recherches en psychologie de la santé (e.g., Breakwell, 2014; Dany, 2015). Les recherches communautaires (Morin, Terrade, Préau, 2012) constituent également des «laboratoires » pour penser l'intervention publique en termes de santé.

(5) Susciter la reconnaissance et l'utilisation des différents types de savoirs associés à la santé et à la maladie

Confrontés aux enjeux de la santé et de la maladie, les individus sont à même de vivre des formes de « rationalités » différentes ayant chacune leurs logiques et fonctions propres. La recherche sur ces différents modes de connaissance de la réalité montre qu'ils ne sont ni juxtaposés, ni en alternance mécanique, ni a fortiori en opposition, leur coexistence puise dans la dynamique du lien social et de la maîtrise des situations (cf. Apostolidis, Dany, 2012). Cette forme de pensée mobilise de manière composite et polyphasique des modalités distinctes de connaissance de la réalité associée à la santé, à la maladie, aux soins ou au risque (e.g. savoirs médicaux, savoirs sociaux, savoirs affectifs, savoirs sensibles) en fonction des interactions, des relations sociales et des situations auxquelles la pensée est confrontée (Morin, 2004). La recherche en psychologie de la

\footnotetext{
${ }^{1}$ «La psychologie communautaire a pour objet la production de changement social [...] par la participation des communautés, dans le but de les rendre dépositaire du pouvoir social et du contrôle de leur environnement » (Montero, Diaz, 2007).
} 
santé peut participer, de fait, à «dépasser» certaines oppositions (rationnel versus irrationnel, scientifique versus profane). Ainsi, la recherche communautaire, par la reconnaissance explicite des savoirs profanes permet dans les effets de sa mise en œuvre de bousculer ces oppositions pour permettre des changements de pratiques, croyances et représentations classiquement travaillés en psychologie de la santé (Préau, Lorente, Sagaon-Teyssier, Champenois, Le Gall, Mabire, Spire, Mora, Yazdanpanah, Suzan, 2016 ; Demange, Henry, Préau, 2012).

\section{(6) Questionner de façon critique les fondements et objectifs de la psychologie de la santé}

Cet axe peut être rapproché du courant de la psychologie critique de la santé qui a largement contribué à questionner la psychologie de la santé mainstream et certaines « dérives » positivistes qui peuvent lui être associées (Crosslay, 2000 ; Murray, 2004). Une des critiques majeure porte sur l'accentuation des facteurs de personnalité ou des variables psychologiques qui a pour conséquence une tendance à concevoir un sujet isolé de son contexte (Morin, 2004; Santiago-Delefosse, Chamberlain, 2008). Ce courant est associé de façon privilégiée à l'usage de méthodologies qualitatives, bien que cet usage ne soit pas exclusif à une démarche critique (Smith, Harré, Van Langenhove, 1995). Les travaux engagés dans cette perspective critique visent une approche intégrative des différents niveaux d'explication possibles afin de développer une visée compréhensive de l'expérience de la santé et de la maladie. Les travaux réalisés dans ce domaine ont également pour objectif d'expliciter les valeurs sous-jacentes aux recherches en psychologie de la santé (i.e. la façon dont elles peuvent conforter une idéologie individualisante, les enjeux normatifs associés à l'élaboration des savoirs et outils dans le champ de la psychologie de la santé).

\section{Quelques exemples actuels de recherches en psychologie de la santé}

Tous ces axes peuvent être explorés à travers diverses méthodes et techniques. Des méthodes quantitatives tout comme des méthodes qualitatives sont utilisées en psychologie de la santé soit de manière isolée, soit de manière complémentaire. De multiple outils/techniques peuvent servir pour recueillir les données dans ces différentes recherches: questionnaires, entretiens, focus group, échantillonnage des expériences en vie quotidienne (Experience Sampling Model $^{2}$ ), marqueurs biologiques,... En psychologie de la santé coexistent à la fois (1) recherche fondamentale et (2) recherche interventionnelle.

\section{(1) Recherche fondamentale}

\footnotetext{
${ }^{2}$ La méthode ESM interroge la personne dans sa vie quotidienne de manière répétée dans un temps limité (par exemple 3 fois par jour pendant 5 jours). Elle permet ainsi d'obtenir des évaluations brèves, prospectives et compréhensives des états mentaux et des comportements des individus.
} 
Depuis de nombreuses années, des travaux multidisciplinaires visent à comprendre l'apparition et l'évolution de différents problèmes de santé publique (cancers, maladies cardio-vasculaires,...). Dans le champ de la prévention, la recherche menée actuellement par Déborah Loyal, financée par un contrat doctoral ministériel (MESR), a pour objectif principal d'explorer le concept de burnout dans la période périnatale dans un échantillon de 144 femmes en fin de grossesse. Elle évalue notamment les symptômes anxio-dépressifs, le burnout, l'idéologie du maternage intensif, les pratiques de maternage, la satisfaction et la compétence dans le rôle parental à la fois par des méthodes quantitative avec des questionnaires administrés à trois temps (fin de grossesse, 2 mois et 4 mois après l'accouchement) et qualitative avec des entretiens semi-directifs. Ces travaux visent, d'une part, à proposer un modèle alternatif de compréhension des troubles périnataux, moins centré sur des caractéristiques individuelles et davantage sur les demandes, le contexte et les normes associées au rôle maternel et, d'autre part, à améliorer la prévention et la prise en charge des troubles périnataux (Loyal, Sutter, Rascle, 2017). Dans le domaine du vécu du handicap, la recherche menée par Mathilde Carlsberg, financée la Fondation des Gueules Cassées, évalue la qualité de vie ainsi que différents processus psychologiques de patients cérébrolésés participant à un programme d'aide à la réinsertion sociale et professionnelle (Carlsberg, Koleck, Mazaux, Wiart, Coste, Marsollet, Simion, Soriano, 2016). Elle concerne des personnes majeures présentant une lésion cérébrale acquise de nature traumatique, vasculaire ou autre, et susceptibles de présenter une préconisation de réinsertion sociale et/ou professionnelle. Des questionnaires sont complétés par les participants tous les trois mois pendant deux ans lors d'une rencontre avec le psychologue. Ces travaux fourniront une meilleure appréhension de l'évolution de différents facteurs psychologiques au cours du programme d'aide à la réinsertion pour l'améliorer, si nécessaire, en tenant compte au plus près de la réalité et du vécu du patient. Une autre recherche prospective de cette équipe auprès de patients présentant une aphasie suite à un AVC a mis en évidence les effets négatifs de l'état dépressif et des troubles de la communication verbale, évalués au cours du mois suivant l'AVC, sur la qualité de vie ultérieure des patients et souligné ainsi l'importance de prendre en charge de manière précoce ces deux facteurs pour améliorer le bien-être des patients (Koleck, Gana, Lucot, Darrigrand, Mazaux, Glize, 2017).

\section{(2) Recherche interventionnelle}

Les recherches en psychologie de la santé se centrent également sur l'évaluation des prises en charge proposées aux patients afin de déterminer les modalités d'accompagnement les plus adaptées. Dans le champ de l'éducation thérapeutique, la thèse menée par Jérôme Foucaud, il y a quelques années, avait pour objectifs d'élaborer un programme d'éducation thérapeutique en quatre séances à 
destination de patients asthmatiques et d'en évaluer l'efficacité. Les patients asthmatiques ont été répartis dans un groupe expérimental et dans un groupe contrôle. Les connaissances sur l'asthme, la symptomatologie dépressive, le soutien social perçu, le contrôle perçu, les stratégies de coping, l'adhésion thérapeutique, la qualité de vie et le recours aux soins d'urgence ont été évalués par des questionnaires avant les séances, à la fin du programme et à 18 mois. Un entretien sur leur vécu des séances a été proposé aux patients ayant suivi le programme. Les résultats de cette étude soulignent l'impact favorable de l'approche éducative proposée sur la qualité de vie des patients asthmatiques ainsi que sur leur façon de percevoir et de gérer leur maladie. Il se dégage un modèle éducatif structuré, évalué et appropriable par les professionnels de santé (Foucaud, 2010). Dans le domaine de l'oncologie, la recherche menée par Stéphane Faury, financée par l'Institut National du Cancer (INCa), a pour objectif d'évaluer l'impact d'un accompagnement psychologique sur la qualité de vie et le vécu psychosocial de patients atteints d'un cancer du rectum porteurs d'une stomie temporaire. Les patients sont répartis dans un groupe expérimental et dans un groupe contrôle. Ils complètent quatre protocoles de questionnaires (qualité de vie, honte liée à l'image du corps, partage social des émotions, perspective temporelle,...) avant et un mois après la création de la stomie, un et trois mois après la fermeture de la stomie. Les résultats de cette recherche fourniront des pistes pour penser un accompagnement interdisciplinaire afin d'améliorer le parcours de soins des patients atteints d'un cancer du rectum avant, pendant et après la stomie, notamment sur le plan de l'accompagnement psychosocial (Faury, Rullier, Quintard, 2016). Dans d'autres contextes comme celui de la prise en charge du VIH, ce sont davantage les enjeux de prévention de comportements considérés comme à risque qui sont explorés. Ainsi, l'approche classique de prévention a pu être bousculée par les travaux menés en psychologie de la santé autour de la prévention dite combinée qui consiste à proposer différents outils de prévention dont on va chercher à appréhender en quoi des déterminants psychologiques, psychosociaux et contextuels vont impacter les pratiques des individus. L'essai ANRS-IPERGAY mené en France depuis 2011, qui a montré l'efficacité de la prévention médicalisée du VIH, a ainsi permis de mettre en évidence l'intérêt d'une approche communautaire face au public des hommes ayant des relations sexuelles avec des hommes qui ont des prises de risque répétées, en intégrant les recueils de données relatives à l'univers de croyances de ces individus. Dans cette étude qui a mobilisé différentes approches disciplinaires, la psychologie de la santé a pu mettre en avant l'intérêt des approches méthodologiques de triangulation, mais aussi des approches théoriques permettant de comprendre les croyances et les comportements des divers acteurs concernés (Mabire, 2015).

\section{Conclusion :}

La recherche en psychologie de la santé présente de multiples facettes et nécessite un travail en 
collaboration entre patients, cliniciens et chercheurs. Quelle qu'en soit sa forme, elle doit toujours conserver comme objectif premier de maintenir et/ou d'améliorer le bien-être de la personne. Ses apports doivent être utiles à la fois pour le patient, ses proches, les professionnels de santé. La question primordiale à l'heure actuelle est sans doute celle de l'articulation entre le recherche et la pratique : comment psychologues de terrain et chercheurs en psychologie de la santé peuvent-ils mieux communiquer pour que les uns puissent faire d'un constat de terrain une question de recherche et que les autres puissent s'approprier et utiliser les résultats de la recherche en psychologie de la santé dans leur pratique quotidienne?

\section{Bibliographie}

Ajzen J., 1991, " The theory of planned behaviour », Organizational Behavior and Human Decision Processes, 50 : 179-211.

Apostolidis T., Dany L., 2012, «Pensée sociale et risques dans le domaine de la santé : le regard des représentations sociales », Psychologie Française, $57:$ 67-81.

Ajzen I., Fishbein M., 1980, « The influence of attitudes on behavior», in Albarracin D., Johnson B. T., Zanna M. P. (sous la direction de), The handbook of attitudes. Mahwah : Erlbaum.

Brashers D. E., Goldsmith, D., 2009, Communicating to manage health and illness. New York, Routledge.

Breakwell, G. M., 2014, The psychology of risk. Cambridge, Cambridge University Press.

Bruchon-Schweitzer M., Boujut E., 2014, Psychologie de la santé : concepts, méthodes et modèles. Paris, Dunod.

Bruchon-Schweitzer M., Quintard B., 2001, Personnalités et maladies. Stress, coping et ajustement. Paris, Dunod.

Carlsberg M., Koleck M., Mazaux JM., Wiart L., Coste E., Marsollet H., Simion A., Soriano V., 2016, « Stratégies de coping, qualité de vie et lésions cérébrales ». Communication orale présentée au $9^{\text {ème }}$ Congrès Francophone de Psychologie de la Santé, Lyon, France.

Corraze J., 1992, Psychologie et médecine. Paris, Presses Universitaires de France.

Crossley M., 2000, Rethinking health psychology. Buckingham, McGraw-Hill.

Demange E., Henry E., Préau M., 2012, De la recherche en collaboration à la recherche communautaire. Un guide méthodologique (From collaborative research to community-based research. A methodological toolkit). Paris, ANRS/Coalition Plus.

Dany L., 2015, «Prévention entre continuité et changements : réflexions psychosociales », in Saint-Jean M., Péoc'h N., Bastiani B. (sous la direction de), Accompagner le changement dans le 
champ de la santé. Paris, De Boeck-Estem.

Faury S., Rullier E., Quintard B., 2016, « Image du corps, dépression et anxiété des patients atteints d'un cancer du rectum avec stomie temporaire : une étude exploratoire longitudinale ». Ann Med Psychol, Doi : 10.1016/j.amp.2016.02.019.

Fisher G. N., 2004, Traité de psychologie de la santé. Paris, Dunod.

Fischer G. N., Tarquinio, C., 2014, Les concepts fondamentaux de la psychologie de la santé.

Paris, Dunod.

Fischer G. N., Tarquinio C., 2014, Psychologie de la santé : applications et interventions. Paris, Dunod.

Foucaud J., 2010, «Intervention éducative menée auprès de patients asthmatiques suivis sur 18 mois » in Foucaud J., Bury J., Balcou-Debussche M., Eymard C. (sous la direction de). Éducation thérapeutique du patient. Modèles, pratiques et évaluation. Saint-Denis: INPES.

Koleck M., Bruchon-Schweitzer M., Bourgeois, M. L., 2003, « Stress et coping : un modèle intégratif en psychologie de la santé », Annales Médico-psychologiques, 161 : 809-815.

Koleck M., Gana K., Lucot C., Darrigrand B., Mazaux JM., Glize B., 2017, « Quality of Life in Aphasic Patients One Year after a First-Stroke ", Quality of Life Research. 26 : 45-54.

Lazarus R. S., Folkman S., 1984, Stress, Appraisal, and Coping. New York, Springer.

Leroy T., Fournier E., Penel N., Christophe V., 2016, «Crossed views of burden and emotional distress of cancer patients and family caregivers during palliative care $»$. Psychooncology, $25: 1278-1285$.

Loyal D., Sutter A.-L., Rascle N., 2017, « Mothering Ideology and Work Involvement in Late Pregnancy: A Clustering Approach». Journal of Child and Family Studies, 1-15. https://doi.org/10.1007/s10826-017-0786-

Mabire X., Laguette V., Spire B., Suzan-Monti M., Lorente N., Girard G., Trachman M., Molina J-M., Capitant C., Mora M., Rojas-Castro D., Préau M., 2015, « Longitudinal qualitative study: what do participants experience during the ANRS-Ipergay trial ? » Communication orale présentée à la 12th AIDSImpact International Conference, Amsterdam, Neitherlands.

Matarazzo, J. D., 1980, « Behavioral health and behavioral medicine », American Psychologist, $35: 807-817$.

Montero M., Diaz N., 2007, «Latin american community psychology: development, implications, and challenges within a social change agenda », in Reich S., Riemer M., Prilleltensky I., Montero M. (sous la direction de), International Community Psychology. Springer, New York.

Morin M., 2008, Parcours de santé, Paris, Armand Colin. 
Morin M., Terrade F., Préau M., 2012, « Psychologie communautaire et psychologie de la santé: l'implication de la recherche psychosociale dans la promotion de la santé », Psychologie Française, 57 : 111-118.

Murray M., 2014, Critical health psychology. New York, Palgrave Macmillan.

Muñoz Sastre M. T., Mullet E., Sorum, P. C., 2011, « Breaking bad news: The patients' view », Health Communication, 26 : 649-655.

Ogden J., 2008, Psychologie de la santé, Bruxelles, De Boeck.

Pédinielli J. L., 1999, « Les « théories » personnelles des patients », Pratiques Psychologiques, $4: 53-62$.

Petrie K. J., Weinman J. A., 1997, Perception of health and illness. Amsterdam, Harwood. Préau M., Morin M., 2005, «L'évaluation psychosociale de la qualité de vie des personnes infectées par le VIH », Pratiques psychologiques, 11 : 387-402.

Préau M., Beaulieu-Prévost D., Henry E., Veillette-Bourbeau L., Otis J., 2015, « HIV serostatus disclosure: development and validation of indicators considering target and modality. Results from a community-based research in 5 countries », Social Science and Medicine, $146: 137-146$.

Préau M., Lorente N., Sagaon-Teyssier L., Champenois K., Le Gall JM., Mabire X., Spire B., Mora M., Yazdanpanah Y., Suzan M., 2016, « Factors associated with satisfaction with community-based non-medicalized counseling and testing using HIV rapid tests among MSM in France ", Aids Care, 28 : 1240-1248.

Radley, A., 1994, Making sense of illness. London, Sage.

Rogers R. W., 1975, «A protection motivation theory of fear appeals and attitude change », Journal of Psychology, 91 : 93-114.

Rosenstock I. M., 1974, « The health belief model and preventive health behavior ». Health Education Monograph, 2 : 35-86.

Rutter D., Quine, L., 2002, Changing health behaviour. Buckingham, Open University Press.

Sarafino E. P., 1990, Health psychology. New York, Wiley.

Smith J. A., Harré R., Van Langenhove L., 1995, Rethinking methods in psychology. London, Sage.

Taylor S. E., 1991, Health psychology. New York, McGraw-Hill. 
